Pacific

Journal of

Mathematics

ON WHITTAKER MODULES FOR A LIE ALGEBRA ARISING FROM THE 2-DIMENSIONAL TORUS

Shaobin Tan, Qing Wang And Chengkang Xu 


\title{
ON WHITTAKER MODULES FOR A LIE ALGEBRA ARISING FROM THE 2-DIMENSIONAL TORUS
}

\author{
ShaObin TAN, QING WANG AND ChengKang XU
}

\begin{abstract}
Let $A$ be the ring of Laurent polynomials in two variables and $B$ be the set of skew derivations of $A$. We denote by $\tilde{L}$ the semidirect product of $A$ and $B$, and by $L$ the universal central extension of the derived Lie algebra of $\tilde{L}$. We study the Whittaker modules for the Lie algebra $L$. The irreducibilities for the universal Whittaker modules are given. Moreover, a $\mathbb{Z}$-gradation is defined on the universal Whittaker modules and we determine all $\mathbb{Z}$-graded irreducible quotients of the reducible universal Whittaker modules.
\end{abstract}

\section{Introduction}

The Lie algebra we considered in this paper can be seen as a generalization of the rank one Heisenberg-Virasoro algebra. The rank one Heisenberg-Virasoro algebra HVir was first given in [Arbarello et al. 1988]; it is the universal central extension of the Lie algebra $\mathscr{D}$ of differential operators on a circle of order at most one; $\mathscr{D}$ has a basis $\left\{t^{n}, d_{n}=t^{n+1} d / d t \mid n \in \mathbb{Z}\right\}$ with Lie bracket relations

$$
\left[t^{n}, t^{m}\right]=0, \quad\left[d_{i}, t^{n}\right]=n t^{i+n}, \quad\left[d_{i}, d_{j}\right]=(j-i) d_{i+j},
$$

and HVir has the Lie bracket relations

$$
\begin{aligned}
& {\left[d_{m}, d_{n}\right]=(n-m) d_{m+n}+\delta_{m+n, 0} \frac{m^{3}-m}{12} c_{1},} \\
& {\left[d_{m}, t^{n}\right]=n t^{m+n}+\left(m^{2}-m\right) \delta_{m+n, 0} c_{2},} \\
& {\left[t^{m}, t^{n}\right]=m \delta_{m+n, 0} c_{3},} \\
& {\left[c_{i}, \mathrm{HVir}\right]=0, \quad i=1,2,3 .}
\end{aligned}
$$

One can see that HVir contains a Heisenberg subalgebra and a Virasoro subalgebra. In [Xue et al. 2006], the authors generalized the rank one Heisenberg-Virasoro

Tan was partially supported by NSF of China (grant number 11471268). Wang was partially supported by NSF of China (grant number 11371024), Natural Science Foundation of Fujian Province (grant number 2013J01018) and Fundamental Research Funds for the Central University (grant number 2013121001).

MSC2010: primary 06B15; secondary 17B65, $17 \mathrm{~B} 66$.

Keywords: Whittaker module, infinite-dimensional Lie algebra, torus. 
algebra to the rank two case. More precisely, let $A=\mathbb{C}\left[t_{1}^{ \pm 1}, t_{2}^{ \pm 1}\right]$ be the ring of Laurent polynomials and $B$ be the set of skew derivations of $A$ spanned by elements of the form

$$
E(\alpha)=t^{\alpha}\left(\alpha(2) d_{1}-\alpha(1) d_{2}\right)
$$

where $\alpha=(\alpha(1), \alpha(2)) \in \mathbb{Z}^{2}, t^{\alpha}=t_{1}^{\alpha(1)} t_{2}^{\alpha(2)}$ and $d_{1}, d_{2}$ are degree derivations of $A$. Set $\tilde{L}=A \oplus B$. Then $\tilde{L}$ becomes a Lie algebra under the Lie bracket relations

$$
\left[t^{\alpha}, t^{\beta}\right]=0, \quad\left[t^{\alpha}, E(\beta)\right]=\operatorname{det}\left(\begin{array}{c}
\beta \\
\alpha
\end{array}\right) t^{\alpha+\beta}, \quad[E(\alpha), E(\beta)]=\operatorname{det}\left(\begin{array}{c}
\beta \\
\alpha
\end{array}\right) E(\alpha+\beta),
$$

where $\alpha, \beta \in \mathbb{Z}^{2}$, and

$$
\operatorname{det}\left(\begin{array}{c}
\beta \\
\alpha
\end{array}\right)=\beta(1) \alpha(2)-\alpha(1) \beta(2) .
$$

Let $\tilde{L}^{\prime}$ be the derived Lie subalgebra of $\tilde{L}$. Then $\tilde{L}^{\prime}$ is perfect and has a universal central extension $L$ with the following Lie bracket relations [Xue et al. 2006]:

$$
\begin{aligned}
& {\left[t^{\alpha}, t^{\beta}\right]=0, \quad\left[K_{i}, L\right]=0 \quad \text { for } i=1,2,3,4,} \\
& {\left[t^{\alpha}, E(\beta)\right]=\operatorname{det}\left(\begin{array}{c}
\beta \\
\alpha
\end{array}\right) t^{\alpha+\beta}+\delta_{\alpha+\beta, 0} h(\alpha),} \\
& {[E(\alpha), E(\beta)]=\operatorname{det}\left(\begin{array}{c}
\beta \\
\alpha
\end{array}\right) E(\alpha+\beta)+\delta_{\alpha+\beta, 0} f(\alpha),}
\end{aligned}
$$

where $\alpha, \beta \in \mathbb{Z}^{2} \backslash\{(0,0)\}, K_{1}, K_{2}, K_{3}, K_{4}$ are central elements, and

$$
h(\alpha)=\alpha(1) K_{1}+\alpha(2) K_{2} \quad \text { and } \quad f(\alpha)=\alpha(1) K_{3}+\alpha(2) K_{4} .
$$

One can see that $L$ contains a Virasoro-like subalgebra spanned by

$$
\left\{E(\alpha), K_{3}, K_{4} \mid \alpha \in \mathbb{Z}^{2} \backslash\{(0,0)\}\right\},
$$

which was introduced by Kirkman, Procesi and Small [Kirkman et al. 1994]. In this paper, we study Whittaker modules for the Lie algebra $L$.

Whittaker modules were first discovered by Arnal and Pinczon [1974] in the study of the irreducible representations of $\mathfrak{s l}_{2}(\mathbb{C})$. Kostant [1978] introduced the term "Whittaker module" and studied Whittaker modules for a complex semisimple Lie algebra g. In particular, he built up a one-to-one correspondence between the set of all equivalence classes of Whittaker modules and the set of all ideals in the center of the universal enveloping algebra of $\mathfrak{g}$. Moreover, Whittaker modules were shown to be one important class in the classification of the irreducible modules for the Lie algebra $\mathfrak{s l}_{2}(\mathbb{C})$ [Block 1981]. Since Kostant's definition of Whittaker module for finite-dimensional semisimple Lie algebras is based on a triangular decomposition [Kostant 1978], it is natural to consider Whittaker modules for other algebras with a triangular decomposition, such as Heisenberg algebras, affine Lie 
algebras, generalized Weyl algebras and the Virasoro algebra, which were studied in [Christodoulopoulou 2008; Benkart and Ondrus 2009; Ondrus and Wiesner 2009], respectively. Recently, Whittaker modules for other infinite-dimensional Lie algebras related to the Virasoro algebra were also studied, such as the rank one Heisenberg-Virasoro algebra [Lu and Zhao 2013], the Schrödinger-Witt algebra [Zhang et al. 2010], and so on. Note that these algebras are of rank one, that is, they are graded by $\mathbb{Z}$.

Motivated by these works, Batra and Mazorchuk [2011] defined a Whittaker pair $(\mathfrak{g}, \mathfrak{n})$ for a Lie algebra $\mathfrak{g}$ and a quasinilpotent subalgebra $\mathfrak{n}$ such that $\mathfrak{n}$ acts locally nilpotent on the adjoint module $\mathfrak{g} / \mathfrak{n}$. They obtained a general setup for the study of Whittaker modules, which includes Lie algebras with triangular decomposition and simple Lie algebras of Cartan type. However, this general theory doesn't work for many exceptions such as the generalized Virasoro algebras [Guo and Liu 2011a], the Virasoro-like algebra $\mathscr{V}$ [Guo and Liu 2011b] and the Lie algebra $L$ considered in this paper. Note that the Virasoro-like algebra $\mathscr{V}$ is of rank two, that is, it is graded by $\mathbb{Z}^{2}$. Therefore, Guo and Liu used a different technique to deal with the Whittaker modules for the Lie algebra $\mathscr{V}$ [ibid.]. We note that the Lie algebra $L$ considered in this paper contains the Virasoro-like Lie algebra $\mathscr{V}$ as a Lie subalgebra, and we will see that the study of Whittaker modules for $L$ is more complicated than that for $\mathscr{V}$.

The paper is organized as follows. In Section 2, we state some facts about total orders on $\mathbb{Z}^{2}$ and give the definition of Whittaker modules for the Lie algebra $L$. In Section 3, we determine all the Whittaker vectors for the universal Whittaker modules. In Section 4, we study irreducibility for the universal Whittaker modules. We define a $\mathbb{Z}$-gradation on the universal Whittaker modules and determine all $\mathbb{Z}$ graded irreducible quotients for the reducible universal Whittaker modules. Finally, we prove some more properties of these $\mathbb{Z}$-graded irreducible quotients.

Throughout this paper, we denote the sets of complex numbers, nonzero complex numbers, integers, nonnegative integers and positive integers by $\mathbb{C}, \mathbb{C}^{\times}, \mathbb{Z}, \mathbb{Z}_{+}$ and $\mathbb{N}$, respectively. All Lie algebras mentioned in this paper are over the complex field $\mathbb{C}$. The universal enveloping algebra for a Lie algebra $\mathfrak{g}$ is denoted by $\mathcal{U}(\mathfrak{g})$.

\section{Whittaker modules for the Lie algebra $L$}

In this section, we recall the definition of the Lie algebra $L$ given in [Xue et al. 2006] and the definition of Whittaker module. We also present some facts about them.

For an element $\alpha$ in $G=\mathbb{Z}^{2}$, we denote $\alpha=(\alpha(1), \alpha(2))$. For any $\alpha, \beta \in \mathbb{Z}^{2}$, we set

$$
\operatorname{det}\left(\begin{array}{c}
\beta \\
\alpha
\end{array}\right)=\beta(1) \alpha(2)-\alpha(1) \beta(2)
$$


For any $\alpha \in G \backslash\{\boldsymbol{0}=(0,0)\}$, let $X(\alpha)$ denote $t^{\alpha}$ or $E(\alpha)$ if it has no special explanation. The Lie algebra $L$ is spanned by the elements of the form

$$
\left\{t^{\alpha}, E(\alpha), K_{i} \mid \alpha \in \mathbb{Z}^{2} \backslash\{\mathbf{0}\}, i=1,2,3,4\right\},
$$

with Lie bracket relations defined by (1-1). Clearly, $L$ is $\mathbb{Z}^{2}$-graded and contains a Virasoro-like algebra $\mathscr{V}$ as the Lie subalgebra spanned by

$$
\left\{E(\alpha), K_{3}, K_{4} \mid \alpha \in \mathbb{Z}^{2} \backslash\{\mathbf{0}\}\right\} .
$$

Fix a total order $\prec$ on $G=\mathbb{Z}^{2}$ which is compatible with the addition of $G$, i.e., $\alpha \prec \beta$ implies $\alpha+\gamma \prec \beta+\gamma$ for all $\gamma \in G$. We have the obvious meanings for $\preceq, \succ$, and $\succeq$. Then we have a decomposition $G=G_{+} \uplus\{\boldsymbol{0}\} \uplus G_{-}$, where $G_{ \pm}=\{\alpha \in G \mid \pm \alpha \succ \mathbf{0}\}$.

We say that $\prec$ is dense if for any $\alpha \in G_{+}$, there is some $\beta \in G_{+}$such that $\beta \prec \alpha$; $\prec$ is discrete if there exists a smallest element in $G_{+}$. For example, the lexicographical order is discrete, since $(0,1)$ is the smallest element in $G_{+}$. Dense total orders on $G$ exist. For example, let $\alpha=(\alpha(1), \alpha(2)), \beta=(\beta(1), \beta(2))$. We say $\alpha \prec \beta$ if $\alpha(1)+\alpha(2) \pi<\beta(1)+\beta(2) \pi$. One can check that this is a dense compatible total order on $G$. The following lemma is from [Guo and Liu 2011b].

Lemma 2.1. (1) Nonzero elements $\alpha, \beta \in G$ form a basis of $G$ if and only if $\operatorname{det}\left(\begin{array}{l}\alpha \\ \beta\end{array}\right)= \pm 1$.

(2) If $\prec$ is dense, then for any $\alpha \succ \mathbf{0}$, there is some $\mathbf{0} \prec \beta \prec \alpha$ such that $\operatorname{det}\left(\begin{array}{l}\alpha \\ \beta\end{array}\right) \neq 0$.

(3) If $\prec$ is discrete, let $\epsilon$ denote the smallest positive element in $G$. Then there exists $\epsilon^{\prime} \succ \mathbf{0}$ such that $\epsilon, \epsilon^{\prime}$ form a basis of $G$.

According to the total order on $G$ fixed above, $L$ has a triangular decomposition

$$
L=L_{-} \oplus L_{0} \oplus L_{+}
$$

where $L_{ \pm}=\operatorname{Span}_{\mathbb{C}}\left\{t^{\alpha}, E(\alpha) \mid \pm \alpha \succ \mathbf{0}\right\}$ and $L_{0}=\operatorname{Span}_{\mathbb{C}}\left\{K_{i} \mid i=1,2,3,4\right\}$.

Recall from [Batra and Mazorchuk 2011] that a Lie algebra $\mathfrak{g}$ is called quasinilpotent if

$$
\bigcap_{k \in \mathbb{N}} \mathfrak{g}^{k}=0
$$

where $\mathfrak{g}^{k+1}=\left[\mathfrak{g}^{k}, \mathfrak{g}\right]$ is defined by induction. Now we claim that $L_{+}$is not quasinilpotent. Indeed, $L_{+}$contains $\mathscr{V}_{+}=\bigoplus_{\alpha \in G_{+}} \mathbb{C} E(\alpha)$ as a subalgebra, which is proved to be not quasinilpotent in [Guo and Liu 2011b], so that

$$
\bigcap_{k \in \mathbb{N}} L_{+}^{k} \supseteq \bigcap_{k \in \mathbb{N}} \mathscr{V}_{+}^{k} \neq 0
$$


So $\left(L, L_{+}\right)$is not a Whittaker pair in the sense of [Batra and Mazorchuk 2011], and the general theory for Whittaker modules there does not apply to the Lie algebra $L$. Thus we treat it as follows.

Fix any nonzero Lie algebra homomorphism $\varphi: L_{+} \rightarrow \mathbb{C}$ and let $k_{1}, k_{2}, k_{3}, k_{4} \in \mathbb{C}$. Given an $L$-module $V$, a vector $v \in V$ is called a Whittaker vector of type $\left(\varphi, k_{1}, k_{2}, k_{3}, k_{4}\right)$ if $x v=\varphi(x) v$ for all $x \in L_{+}$, and $K_{i} v=k_{i} v$ for $i=1,2,3,4$. $V$ is called a Whittaker module of type $\left(\varphi, k_{1}, k_{2}, k_{3}, k_{4}\right)$ if $V=U(L) v$ for some Whittaker vector $v$ of type $\left(\varphi, k_{1}, k_{2}, k_{3}, k_{4}\right)$. In this paper, all Whittaker modules and Whittaker vectors are of type $\left(\varphi, k_{1}, k_{2}, k_{3}, k_{4}\right)$ if not specified. Clearly, $u$ is a Whittaker vector if and only if $(X(\alpha)-\varphi(X(\alpha))) u=0$ for all $\alpha \in G_{+}, X(\alpha)=t^{\alpha}$ and $E(\alpha)$. Notice that $\varphi\left(L_{+}^{2}\right)=\left[\varphi\left(L_{+}\right), \varphi\left(L_{+}\right)\right]=0$. We have the following facts.

Proposition 2.2. Let $\prec$ be a total order on $G$.

(1) If $\prec$ is dense, then any Lie algebra homomorphism $\varphi: L_{+} \rightarrow \mathbb{C}$ is the zero homomorphism.

(2) If $\prec$ is discrete and $\epsilon$ denotes the smallest positive element in $G$, then $\varphi\left(t^{\alpha}\right)=$ $\varphi(E(\alpha))=0$ for all $\alpha \in G_{+} \backslash \mathbb{Z} \epsilon$.

Proof. (1) Suppose $\prec$ is dense. Then by Lemma 2.1, for any $\alpha \in G_{+}$there is some $\beta \in G_{+}$such that $\beta \prec \alpha$ and $\operatorname{det}\left(\begin{array}{l}\alpha \\ \beta\end{array}\right) \neq 0$. Thus

$$
X(\alpha)=\frac{1}{\operatorname{det}\left(\begin{array}{c}
\beta \\
\alpha-\beta
\end{array}\right)}[E(\alpha-\beta), X(\beta)]=\frac{1}{\operatorname{det}\left(\begin{array}{c}
\beta \\
\alpha
\end{array}\right)}[E(\alpha-\beta), X(\beta)] \in L_{+}^{2} .
$$

So $\varphi\left(t^{\alpha}\right)=\varphi(E(\alpha))=0$, and this shows that $\varphi=0$.

(2) Let $\alpha \in G_{+} \backslash \mathbb{Z} \epsilon$. We have $\alpha-i \epsilon \in G_{+} \backslash \mathbb{Z} \epsilon$ for all $i \in \mathbb{Z}$, and $\operatorname{det}\left(\begin{array}{l}\alpha \\ \epsilon\end{array}\right) \neq 0$. Thus

$$
X(\alpha)=\frac{1}{\operatorname{det}\left(\begin{array}{c}
\alpha-\epsilon \\
\epsilon
\end{array}\right)}[X(\epsilon), E(\alpha-\epsilon)]=\frac{1}{\operatorname{det}\left(\begin{array}{c}
\alpha \\
\epsilon
\end{array}\right)}[X(\epsilon), E(\alpha-\epsilon)] \in L_{+}^{2},
$$

which shows that $\varphi\left(t^{\alpha}\right)=\varphi(E(\alpha))=0$ for all $\alpha \in G_{+} \backslash \mathbb{Z} \epsilon$.

Thus, we assume that $\prec$ is discrete with smallest positive element $\epsilon$ in $G$ throughout the rest of this paper.

\section{Whittaker vectors in universal Whittaker modules}

In this section we study the universal Whittaker module and determine all its Whittaker vectors. By Lemma 2.1, we know that there exists $\epsilon^{\prime} \in G_{+}$such that $\left\{\epsilon, \epsilon^{\prime}\right\}$ is a basis of $G$. We will always use this basis for $G$ from now on.

We construct the universal Whittaker module of type $\left(\varphi, k_{1}, k_{2}, k_{3}, k_{4}\right)$ over $L$, denoted $M_{\varphi, k_{1}, k_{2}, k_{3}, k_{4}}$, as follows: let $\mathbb{C} \tilde{v}$ be the one-dimensional $\left(L_{0} \oplus L_{+}\right)$-module 
defined by $x \tilde{v}=\varphi(x) \tilde{v}$ for any $x \in L_{+}$and $K_{i} \tilde{v}=k_{i} \tilde{v}$ for $i=1,2,3$, 4. Set

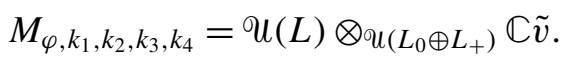

This is a left $U(L)$-module under left multiplication. Set $v=1 \otimes \tilde{v}$. We have $M_{\varphi, k_{1}, k_{2}, k_{3}, k_{4}}=U(L) v$. It is obvious that $M_{\varphi, k_{1}, k_{2}, k_{3}, k_{4}}$ has the following universal property: for any Whittaker module $W$ of type $\left(\varphi, k_{1}, k_{2}, k_{3}, k_{4}\right)$ generated by a Whittaker vector $w$, there is an $L$-module epimorphism $\phi$ from $M_{\varphi, k_{1}, k_{2}, k_{3}, k_{4}}$ to $W$ which maps $v$ to $w$.

By the Poincaré-Birkhoff-Witt (PBW) theorem, $M_{\varphi, k_{1}, k_{2}, k_{3}, k_{4}}$ is isomorphic to $U\left(L_{-}\right)$as a vector space. Let $L_{-}=L_{-}^{t} \oplus L_{-}^{E}$, where

$$
L_{-}^{t}=\operatorname{Span}_{\mathbb{C}}\left\{t^{-\alpha} \mid \alpha \succ 0\right\}, \quad L_{-}^{E}=\operatorname{Span}_{\mathbb{C}}\{E(-\alpha) \mid \alpha \succ 0\} .
$$

Since $U\left(L_{-}^{t}\right)$ and $U\left(L_{-}^{E}\right)$ have $\mathbb{C}$-bases

$$
B^{t}=\left\{1, t^{-\beta_{m}} \cdots t^{-\beta_{1}} \mid m \in \mathbb{N}, \beta_{m} \succeq \cdots \succeq \beta_{1} \succ 0\right\}
$$

and

$$
B^{E}=\left\{1, E\left(-\alpha_{n}\right) \cdots E\left(-\alpha_{1}\right) \mid n \in \mathbb{N}, \alpha_{n} \succeq \cdots \succeq \alpha_{1} \succ 0\right\},
$$

respectively, $\mathcal{U}\left(L_{-}\right)$has a $\mathbb{C}$-basis

$$
\begin{aligned}
B=B^{t} B^{E}=B^{t} \cup B^{E} \cup\left\{t^{-\beta_{m}} \cdots t^{-\beta_{1}} E\left(-\alpha_{n}\right) \cdots E\left(-\alpha_{1}\right)\right. \\
\\
\left.\mid m, n \in \mathbb{N}, \alpha_{n} \succeq \cdots \succeq \alpha_{1} \succ 0, \beta_{m} \succeq \cdots \succeq \beta_{1} \succ 0\right\}
\end{aligned}
$$

and $M_{\varphi, k_{1}, k_{2}, k_{3}, k_{4}}$ has a $\mathbb{C}$-basis $B v$. For convenience, we set $M=M_{\varphi, k_{1}, k_{2}, k_{3}, k_{4}}$ and

$$
\begin{array}{ll}
E_{ \pm}=\bigoplus_{k \in \mathbb{N}} \mathbb{C} E( \pm k \epsilon), & T_{ \pm}=\bigoplus_{k \in \mathbb{N}} \mathbb{C} t^{ \pm k \epsilon}, \\
H_{ \pm}=E_{ \pm} \oplus T_{ \pm}, & H=H_{-} \oplus L_{0} \oplus H_{+}, \\
E=E_{-} \oplus E_{+}, & T=T_{-} \oplus T_{+} .
\end{array}
$$

Set

$$
M(H)=U(H) v=U\left(H_{-}\right) v, \quad M(T)=U(T) v=U\left(T_{-}\right) v .
$$

For $\alpha \in G$, set $\alpha=\alpha[1] \epsilon+\alpha[2] \epsilon^{\prime}$, where $\alpha[1], \alpha[2] \in \mathbb{Z}$.

Lemma 3.1. (1) If $\alpha \in G_{+}$, then $\alpha[2] \geq 0$. In particular, if $\alpha \in G_{+} \backslash \mathbb{Z} \epsilon$, then $\alpha[2]>0$.

(2) If $\alpha \in G_{+} \backslash \mathbb{Z} \epsilon$, then for any $u \in M(H), x \in \mathcal{U}\left(L_{-}\right)$, we have

$$
(X(\alpha)-\varphi(X(\alpha))) x u=[X(\alpha), x] u .
$$

(3) Let $\alpha_{1}, \ldots, \alpha_{n} \in G_{+}, \alpha \in G_{+} \backslash \mathbb{Z} \epsilon$. If $\alpha-\sum_{i=1}^{n} \alpha_{i} \in G_{+} \backslash \mathbb{Z} \epsilon$, then we have

$$
X(\alpha) X\left(-\alpha_{n}\right) \cdots X\left(-\alpha_{1}\right) w=0 \quad \text { for } w \in M(H),
$$


where all $X(\beta)$ denote $t^{\beta}$ or $E(\beta)$.

Proof. (1) Suppose $\alpha$ [2] $<0$. Then we have $-\alpha[2] \epsilon^{\prime} \succeq \epsilon^{\prime} \succ \alpha[1] \epsilon$, which implies $\alpha=\alpha[1] \epsilon+\alpha[2] \epsilon^{\prime} \prec 0$. This is a contradiction with $\alpha \in G_{+}$.

(2) We may assume $u=X\left(-n_{1} \epsilon\right) \cdots X\left(-n_{s} \epsilon\right) v$, where $s \in \mathbb{Z}_{+}, n_{1}, \ldots n_{s} \in \mathbb{N}$. Then

$$
\begin{aligned}
(X(\alpha)-\varphi(X(\alpha))) x u & =X(\alpha) x u-x X\left(-n_{1} \epsilon\right) \cdots X\left(-n_{s} \epsilon\right) X(\alpha) v \\
& =[X(\alpha), x] u+x\left[X(\alpha), X\left(-n_{1} \epsilon\right) \cdots X\left(-n_{s} \epsilon\right)\right] v .
\end{aligned}
$$

Notice that

$$
x\left[X(\alpha), X\left(-n_{1} \epsilon\right) \cdots X\left(-n_{s} \epsilon\right)\right] v \in x \sum_{\eta \in G_{+} \backslash \mathbb{Z} \epsilon} u(H) X(\eta) v=0
$$

by Proposition 2.2, and thus (3-1) holds.

(3) Now we prove (3-2) by induction on $n$. For $n=0$, since $\alpha \in G_{+} \backslash \mathbb{Z} \epsilon$, we have that $\varphi(X(\alpha))=0$ by Proposition 2.2. Hence, by (2),

$$
X(\alpha) w=(X(\alpha)-\varphi(X(\alpha))) w=[X(\alpha), 1] w=0
$$

for $w \in M(H)$. Suppose that $n>0$ and that the result holds for any positive integer $k<n$. Then for $w \in M(H)$, by applying the induction hypothesis, we have

$$
\begin{aligned}
X(\alpha) X\left(-\alpha_{n}\right) \cdots X\left(-\alpha_{1}\right) w & =\operatorname{det}\left(\begin{array}{c}
\alpha \\
\alpha_{n}
\end{array}\right) X\left(\alpha-\alpha_{n}\right) X\left(-\alpha_{n-1}\right) \cdots X\left(-\alpha_{1}\right) w \\
& =0 . \quad+X\left(-\alpha_{n}\right) X(\alpha) X\left(-\alpha_{n-1}\right) \cdots X\left(-\alpha_{1}\right) w
\end{aligned}
$$

For later use, we define some subsets of $B$. Set

$$
B(0)=\left\{1, t^{-\beta_{m}} \cdots t^{-\beta_{1}} \in B \mid m \in \mathbb{N}, \beta_{m} \succeq \cdots \succeq \beta_{1} \in G_{+} \backslash \mathbb{Z} \epsilon\right\} .
$$

For $h \in \mathbb{N}$, set

$$
\begin{aligned}
& B_{E}(h)=\left\{E\left(-\alpha_{n}\right) \cdots E\left(-\alpha_{1}\right) \in B \mid n \in \mathbb{N}, \alpha_{m} \succeq \cdots \succeq \alpha_{1} \in G_{+} \backslash \mathbb{Z} \epsilon, \sum_{i=1}^{n} \alpha_{i}[2]=h\right\}, \\
& B(h)=B(0) B_{E}(h), \quad B(-h)=\varnothing, \quad B^{\prime}(h)=\bigcup_{h^{\prime}<h} B\left(h^{\prime}\right), \quad \bar{B}(h)=B(h) \cup B^{\prime}(h) .
\end{aligned}
$$

For $h \in \mathbb{N}, \beta \in G_{+} \backslash \mathbb{Z} \epsilon$, set

$$
B_{T}(h, \beta)=\left\{t^{-\beta_{m}} \cdots t^{-\beta_{1}} \mid \beta_{m} \succeq \cdots \succeq \beta_{1}=\beta, \sum_{i=1}^{m} \beta_{i}[2]=h\right\}
$$

and

$$
B_{T}(h)=\bigcup_{\beta \in G_{+} \backslash \mathbb{Z} \epsilon} B_{T}(h, \beta) .
$$


Let $\mathscr{H}=\left\{(h, \beta) \mid B_{T}(h, \beta) \neq \varnothing\right\}$, and define a total order $\gg$ on $\mathscr{H}$ by setting

$$
(h, \beta) \gg\left(h^{\prime}, \beta^{\prime}\right) \quad \text { if } h>h^{\prime} \text {, or } h=h^{\prime} \text { and } \beta \prec \beta^{\prime} .
$$

Moreover, we denote

$$
\begin{array}{ll}
B_{T}^{\prime}(h, \beta)=\bigcup_{(h, \beta) \gg\left(h^{\prime}, \beta^{\prime}\right)} B_{T}\left(h^{\prime}, \beta^{\prime}\right), & B_{T}^{\prime}(h)=\bigcup_{h^{\prime}<h} B_{T}\left(h^{\prime}\right), \\
\bar{B}_{T}(h, \beta)=B_{T}(h, \beta) \cup B_{T}^{\prime}(h, \beta), & \bar{B}_{T}(h)=\bigcup_{h^{\prime} \leq h} B_{T}\left(h^{\prime}\right),
\end{array}
$$

and we set $B_{T}(0)=\bar{B}_{T}(0)=\{1\}, B_{T}^{\prime}(0)=\varnothing$. Then one can see that $B(0)=$ $\bigcup_{h \in \mathbb{Z}_{+}} B_{T}(h)$.

Lemma 3.2. (1) For any $u \in M \backslash B(0) M(H)$, there exists $\eta \in G_{+} \backslash \mathbb{Z} \epsilon$ such that

$$
\left(t^{\eta}-\varphi\left(t^{\eta}\right)\right) u \in B(0) M(H) \backslash \mathbb{C} v .
$$

(2) For any $u^{\prime} \in B(0) M(H) \backslash M(H)$, there exist $\gamma_{1}, \ldots, \gamma_{s} \in G_{+}$such that

$$
\left(E\left(\gamma_{s}\right)-\varphi\left(E\left(\gamma_{s}\right)\right)\right) \cdots\left(E\left(\gamma_{1}\right)-\varphi\left(E\left(\gamma_{1}\right)\right)\right) u^{\prime} \in M(H) \backslash \mathbb{C} v .
$$

Proof. (1) Since $u \in M \backslash B(0) M(H)$, there exists $h \in \mathbb{N}$ such that $u \in \bar{B}(h) M(H)$ and $u \notin B^{\prime}(h) M(H)$. Thus, we may write

$$
u=\sum_{x \in B(h)} x v_{x}+\sum_{y \in B^{\prime}(h)} y v_{y}
$$

where $v_{x}, v_{y} \in M(H)$ and both sums are finite, and the elements $x$ are of the form

$$
x=t^{-\beta_{m}} \cdots t^{-\beta_{1}} E\left(-\alpha_{n}\right) \cdots E\left(-\alpha_{1}\right),
$$

with $m \in \mathbb{Z}_{+}, n \in \mathbb{N}, \beta_{m} \succeq \cdots \succeq \beta_{1} \in G_{+} \backslash \mathbb{Z} \epsilon, \alpha_{n} \succeq \cdots \succeq \alpha_{1} \in G_{+} \backslash \mathbb{Z} \epsilon$ and $\sum_{i=1}^{n} \alpha_{i}[2]=h$, where $m=0$ means that $x=E\left(-\alpha_{n}\right) \cdots E\left(-\alpha_{1}\right)$.

Let $\eta \in G$ be such that $\eta[2]=h, \eta-\sum_{i=1}^{n} \alpha_{i} \in-\mathbb{N} \epsilon$ and, for each $\left(\alpha_{n}, \ldots, \alpha_{1}\right)$ in (3-3) associated to an element $x$ appearing in $\sum_{x \in B(h)} x v_{x}$,

$$
\operatorname{det}\left(\begin{array}{c}
\eta-\sum_{j=i+1}^{n} \alpha_{j} \\
\alpha_{i}
\end{array}\right) \neq 0 \quad \text { for } 1 \leq i \leq n-1 \quad \text { and } \quad \operatorname{det}\left(\begin{array}{c}
\eta \\
\alpha_{n}
\end{array}\right) \neq 0
$$

Since the sum $\sum_{x \in B(h)} x v_{x}$ is finite, it is obvious that such an $\eta$ exists. It follows that $\eta \in G_{+} \backslash \mathbb{Z} \epsilon$, thus we have $\bar{u}=\left(t^{\eta}-\varphi\left(t^{\eta}\right)\right) u=t^{\eta} u$ by Proposition 2.2. Note that $t^{\eta}\left(\sum_{y \in B^{\prime}(h)} y v_{y}\right)=0$ by Lemma 3.1(3), thus we have

$$
\bar{u}=\sum_{x \in B(h)} t^{-\beta_{m}} \cdots t^{-\beta_{1}} t^{\eta} E\left(-\alpha_{n}\right) \cdots E\left(-\alpha_{1}\right) v_{x} .
$$


For every summand in $\bar{u}$, by using Lemma 3.1(3), we have

$$
\begin{aligned}
t^{-\beta_{m}} \cdots t^{-\beta_{1}} t^{\eta} E\left(-\alpha_{n}\right) \cdots E\left(-\alpha_{1}\right) v_{x} \\
=t^{-\beta_{m}} \cdots t^{-\beta_{1}}\left[t^{\eta}, E\left(-\alpha_{n}\right)\right] E\left(-\alpha_{n-1}\right) \cdots E\left(-\alpha_{1}\right) v_{x} \\
\quad \quad+t^{-\beta_{m}} \cdots t^{-\beta_{1}} E\left(-\alpha_{n}\right) t^{\eta} E\left(-\alpha_{n-1}\right) \cdots E\left(-\alpha_{1}\right) v_{x} \\
=\operatorname{det}\left(\begin{array}{c}
\eta \\
\alpha_{n}
\end{array}\right) t^{-\beta_{m}} \cdots t^{-\beta_{1}} t^{\eta-\alpha_{n}} E\left(-\alpha_{n-1}\right) \cdots E\left(-\alpha_{1}\right) v_{x} \\
=\operatorname{det}\left(\begin{array}{c}
\eta \\
\alpha_{n}
\end{array}\right) \prod_{i=1}^{n-1} \operatorname{det}\left(\begin{array}{c}
\eta-\sum_{j=i+1}^{n} \alpha_{j} \\
\alpha_{i}
\end{array}\right) t^{-\beta_{m}} \cdots t^{-\beta_{1}} t^{\eta-\sum_{j=1}^{n} \alpha_{j}} v_{x}
\end{aligned}
$$$$
\in B(0) M(H) \backslash \mathbb{C} v \text {. }
$$

This implies that $\bar{u} \in B(0) M(H) \backslash \mathbb{C} v$.

(2) Since $u^{\prime} \in B(0) M(H) \backslash M(H)$, there exists $h \in \mathbb{N}$ and $\beta \in G_{+} \backslash \mathbb{Z} \epsilon$ such that $u^{\prime} \in \bar{B}_{T}(h, \beta) M(H)$ and $u^{\prime} \notin B_{T}^{\prime}(h, \beta) M(H)$. Thus we may write

$$
u^{\prime}=\sum_{x \in B_{T}(h, \beta)} x v_{x}+\sum_{y \in B_{T}^{\prime}(h, \beta)} y v_{y},
$$

where $v_{x}, v_{y} \in M(H)$ and both sums are finite. Then there exists some $n_{0} \in \mathbb{N}$ such that all $v_{x}, v_{y}$ appeared above lie in $U\left(\sum_{i<n_{0}} \mathbb{C} t^{-i \epsilon} \oplus \mathbb{C} E(-i \epsilon)\right) v$.

Take $\gamma_{1}=\beta-n_{0} \epsilon$, so $\operatorname{det}\left(\begin{array}{c}\gamma_{1} \\ \beta\end{array}\right) \neq 0$. We consider $\left(E\left(\gamma_{1}\right)-\varphi\left(E\left(\gamma_{1}\right)\right)\right) u^{\prime}$. First we consider the term $\left(E\left(\gamma_{1}\right)-\varphi\left(E\left(\gamma_{1}\right)\right)\right) x v_{x}$ for $x \in B_{T}(h, \beta)$. We may write

$$
x=t^{-\beta_{m}} \cdots t^{-\beta_{1}}\left(t^{-\beta}\right)^{k},
$$

where $m \in \mathbb{Z}_{+}, \beta_{m} \succeq \cdots \succeq \beta_{1} \succ \beta, k \in \mathbb{N}$ and $\left(k \beta+\sum_{i=1}^{m} \beta_{i}\right)[2]=h$. Then, by Lemma 3.1(2), we have

$$
\begin{aligned}
\left(E\left(\gamma_{1}\right)-\varphi\left(E\left(\gamma_{1}\right)\right)\right) x v_{x} & \\
= & {\left[E\left(\gamma_{1}\right), t^{-\beta_{m}} \cdots t^{-\beta_{1}}\left(t^{-\beta}\right)^{k}\right] v_{x} } \\
= & \sum_{i=1}^{m} \operatorname{det}\left(\begin{array}{c}
\gamma_{1} \\
\beta_{i}
\end{array}\right) t^{-\beta_{m}} \cdots t^{-\beta_{i}+\beta-n_{0} \epsilon} \cdots t^{-\beta_{1}}\left(t^{-\beta}\right)^{k} v_{x} \\
& \quad+\operatorname{det}\left(\begin{array}{c}
\gamma_{1} \\
\beta
\end{array}\right) x^{\prime} t^{-n_{0} \epsilon} v_{x},
\end{aligned}
$$

where $x^{\prime}=k t^{-\beta_{m}} \cdots t^{-\beta_{1}}\left(t^{-\beta}\right)^{k-1}$. Set $M\left(T_{-n_{0}}\right)=u\left(\bigoplus_{k \neq n_{0}} t^{-k \epsilon}\right) u\left(E_{-}\right) v$. Then we see that the first sum in (3-4) lies in $B_{T}(h-\beta[2]) M\left(T_{-n_{0}}\right)$. Thus we have

$$
\left(E\left(\gamma_{1}\right)-\varphi\left(E\left(\gamma_{1}\right)\right)\right) x v_{x} \equiv \operatorname{det}\left(\begin{array}{c}
\gamma_{1} \\
\beta
\end{array}\right) x^{\prime} t^{-n_{0} \epsilon} v_{x}\left(\bmod B_{T}(h-\beta[2]) M\left(T_{-n_{0}}\right)\right) .
$$


Now we consider the term $\left(E\left(\gamma_{1}\right)-\varphi\left(E\left(\gamma_{1}\right)\right)\right) y v_{y}$ for $y \in B_{T}^{\prime}(h, \beta)$. We may write

$$
y=t^{-\beta_{m}} \cdots t^{-\beta_{1}},
$$

where $\beta_{m} \succeq \cdots \succeq \beta_{1} \in G_{+} \backslash \mathbb{Z} \epsilon, \sum_{i=1}^{m} \beta_{i}[2]=h^{\prime} \leq h$. It is clear that $\beta_{i} \succ \beta$ for all $i$ when $h^{\prime}=h$. By Lemma 3.1(2), we have

$$
\begin{aligned}
\left(E\left(\gamma_{1}\right)-\varphi\left(E\left(\gamma_{1}\right)\right)\right) y v_{y} & =\left[E\left(\gamma_{1}\right), t^{-\beta_{m}} \cdots t^{-\beta_{1}}\right] v_{y} \\
& =\sum_{i=1}^{m} \operatorname{det}\left(\begin{array}{c}
\gamma_{1} \\
\beta_{i}
\end{array}\right) t^{-\beta_{m}} \cdots t^{-\beta_{i}+\beta-n_{0} \epsilon} \cdots t^{-\beta_{1}} v_{y} .
\end{aligned}
$$

If $h^{\prime}<h$, it is obvious that

$$
\left(E\left(\gamma_{1}\right)-\varphi\left(E\left(\gamma_{1}\right)\right)\right) y v_{y} \in B_{T}\left(h^{\prime}-\beta[2]\right) M(H) \subseteq B_{T}^{\prime}(h-\beta[2]) M(H) .
$$

If $h^{\prime}=h$, then we have $\beta_{i}-\beta \in G_{+}$for all $1 \leq i \leq m$. If $\beta_{i}-\beta \in G_{+} \backslash \mathbb{Z} \epsilon$, then it is clear that

$$
t^{-\beta_{m}} \cdots t^{-\beta_{i}+\beta-n_{0} \epsilon} \cdots t^{-\beta_{1}} v_{y} \in B_{T}(h-\beta[2]) M\left(T_{-n_{0}}\right) .
$$

If $\beta_{i}-\beta=n_{i} \epsilon \in \mathbb{N} \epsilon$, then

$$
t^{-\beta_{m}} \cdots t^{-\beta_{i}+\beta-n_{0} \epsilon} \cdots t^{-\beta_{1}} v_{y}=t^{-\beta_{m}} \cdots t^{-\beta_{i+1}} t^{-\beta_{i-1}} \cdots t^{-\beta_{1}} t^{\left(n_{i}-n_{0}\right) \epsilon} v_{y},
$$

which also lies in $B_{T}(h-\beta[2]) M\left(T_{-n_{0}}\right)$. Thus we have

$$
\left(E\left(\gamma_{1}\right)-\varphi\left(E\left(\gamma_{1}\right)\right)\right) y v_{y} \in B_{T}^{\prime}(h-\beta[2]) M(H)+B_{T}(h-\beta[2]) M\left(T_{-n_{0}}\right) .
$$

From this discussion, we see that

$$
\left(E\left(\gamma_{1}\right)-\varphi\left(E\left(\gamma_{1}\right)\right)\right) u^{\prime}=\operatorname{det}\left(\begin{array}{c}
\gamma_{1} \\
\beta
\end{array}\right) \sum_{x \in B_{T}(h, \beta)} x^{\prime} t^{-n_{0} \epsilon} v_{x}+u^{\prime \prime}
$$

for some $u^{\prime \prime} \in B_{T}^{\prime}(h-\beta[2]) M(H)+B_{T}(h-\beta[2]) M\left(T_{-n_{0}}\right)$. Note that

$$
\sum_{x \in B_{T}(h, \beta)} x^{\prime} t^{-n_{0} \epsilon} v_{x} \in B_{T}(h-\beta[2]) t^{-n_{0} \epsilon} M(H)
$$

is linearly independent from $u^{\prime \prime}$. This, together with the facts that

$$
\sum_{x \in B_{T}(h, \beta)} x v_{x} \neq 0 \text { and } \operatorname{det}\left(\begin{array}{c}
\gamma_{1} \\
\beta
\end{array}\right) \neq 0
$$

imply

$$
\operatorname{det}\left(\begin{array}{l}
\gamma_{1} \\
\beta
\end{array}\right) \sum_{x \in B_{T}(h, \beta)} x^{\prime} t^{-n_{0} \epsilon} v_{x} \neq 0
$$


In particular, we have $\left(E\left(\gamma_{1}\right)-\varphi\left(E\left(\gamma_{1}\right)\right)\right) u^{\prime} \notin \mathbb{C} v$. Clearly, we have

$$
\left(E\left(\gamma_{1}\right)-\varphi\left(E\left(\gamma_{1}\right)\right)\right) u^{\prime} \in \bar{B}_{T}(h-\beta[2]) M(H) .
$$

Then, repeating this process finitely many times, we can take some $\gamma_{2}, \ldots, \gamma_{s} \in$ $G_{+} \backslash \mathbb{Z} \epsilon, s \in \mathbb{N}$ such that

$$
\left(E\left(\gamma_{s}\right)-\varphi\left(E\left(\gamma_{s}\right)\right)\right) \cdots\left(E\left(\gamma_{1}\right)-\varphi\left(E\left(\gamma_{1}\right)\right)\right) u^{\prime} \in \bar{B}_{T}(0) M(H) \backslash \mathbb{C} v=M(H) \backslash \mathbb{C} v .
$$

This completes the proof.

Lemma 3.3. For $u \in M(H) \backslash \mathbb{C} v$, there exist $r, s \in \mathbb{Z}_{+}, n_{1}, \ldots, n_{s}, m_{1}, \ldots, m_{r} \in \mathbb{N}$ and $A \in \mathbb{C}^{\times}$such that

$$
\begin{aligned}
&\left(E\left(n_{s} \epsilon\right)-\varphi\left(E\left(n_{s} \epsilon\right)\right)\right) \cdots\left(E\left(n_{1} \epsilon\right)-\varphi\left(E\left(n_{1} \epsilon\right)\right)\right) \\
& \cdot\left(t^{m_{r} \epsilon}-\varphi\left(t^{m_{r} \epsilon}\right)\right) \cdots\left(t^{m_{1} \epsilon}-\varphi\left(t^{m_{1} \epsilon}\right)\right) u=A h(\epsilon)^{r+s} v .
\end{aligned}
$$

Proof. First, we may assume $u \notin M(T)$, and we write

$$
u=\sum_{i=1}^{n} a_{i} f_{i} v_{i}
$$

where all $a_{i} \neq 0, v_{i} \in M(T)$ and $f_{i}$ for $1 \leq i \leq n$ are monic monomials with variables from the set $\{E(-j \epsilon) \mid j \in \mathbb{N}\}$. Without loss of generality, we may assume that $f_{1}$ has the maximal degree, and write

$$
f_{1}=E(-\epsilon)^{m_{1}} \cdots E(-r \epsilon)^{m_{r}}, \quad m_{i} \in \mathbb{Z}_{+},
$$

where $m_{1}, \ldots, m_{r}$ are not all zero. For any monomial $g$ with variables from $\{E(-j \epsilon) \mid j \in \mathbb{N}\}$, note that $\left[t^{i \epsilon}, E(j \epsilon)\right]=\delta_{i+j, 0} i h(\epsilon)$ for any $i, j \in \mathbb{Z}$. Then for any $w \in M(T)$, we have

$$
\left(t^{i \epsilon}-\varphi\left(t^{i \epsilon}\right)\right) g w=i h(\epsilon) \partial_{i}^{\prime}(g) w,
$$

where $\partial_{i}^{\prime}(g)$ is the partial derivative of $g$ with respect to $E(-i \epsilon)$. Then by induction on $r$ it is easy to see that

$$
\left(t^{\epsilon}-\varphi\left(t^{\epsilon}\right)\right)^{m_{1}} \cdots\left(t^{r \epsilon}-\varphi\left(t^{r \epsilon}\right)\right)^{m_{r}} f_{i} v_{i}=\delta_{1, i} \prod_{j=1}^{r} m_{j} !(j h(\epsilon))^{m_{j}} v_{1},
$$

where $\delta_{1, i}$ is the Kronecker delta function and $m_{j}$ ! is the factorial of $m_{j}$. So we get

$$
\left(t^{\epsilon}-\varphi\left(t^{\epsilon}\right)\right)^{m_{1}} \cdots\left(t^{r \epsilon}-\varphi\left(t^{r \epsilon}\right)\right)^{m_{r}} u=A_{1} h(\epsilon)^{m_{1}+\cdots+m_{r}} v_{1}
$$


where $A_{1}=a_{1} \prod_{j=1}^{r} m_{j} ! j^{m_{j}} \neq 0$. If $v_{1} \in \mathbb{C} v$, the lemma is clear. Otherwise, $v_{1} \in M(T)$ and $v_{1} \notin \mathbb{C} v$, and we write

$$
v_{1}=\sum_{i=1}^{n} b_{i} g_{i} v \in M(T),
$$

where all $b_{i} \neq 0$ and $g_{i}$ for $1 \leq i \leq n$ are monic monomials with variables from the set $\left\{t^{-j \epsilon} \mid j \in \mathbb{N}\right\}$. Without loss of generality, we may assume that $g_{1}$ has the maximal degree, and write

$$
g_{1}=\left(t^{-\epsilon}\right)^{n_{1}} \cdots\left(t^{-s \epsilon}\right)^{n_{s}}, n_{i} \in \mathbb{Z}_{+},
$$

where $n_{1}, \ldots, n_{s}$ are not all zero. For any monomial $g$ with variables from the set $\left\{t^{-j \epsilon} \mid j \in \mathbb{N}\right\}$, we have

$$
(E(i \epsilon)-\varphi(E(i \epsilon))) g v=i h(\epsilon) \partial_{i}^{\prime \prime}(g) v,
$$

where $\partial_{i}^{\prime \prime}(g)$ is the partial derivative of $g$ with respect to $t^{-i \epsilon}$. Then by induction on $s$ it is easy to see that

$$
(E(\epsilon)-\varphi(E(\epsilon)))^{n_{1}} \cdots(E(s \epsilon)-\varphi(E(s \epsilon)))^{n_{s}} g_{i} v=\delta_{1, i} \prod_{j=1}^{s} n_{j} !(j h(\epsilon))^{n_{j}} v .
$$

Thus we get

$$
(E(\epsilon)-\varphi(E(\epsilon)))^{n_{1}} \cdots(E(s \epsilon)-\varphi(E(s \epsilon)))^{n_{s}} v_{1}=A_{2} h(\epsilon)^{n_{1}+\cdots+n_{s}} v,
$$

where $A_{2}=b_{1} \prod_{j=1}^{s} n_{j} ! j^{n_{j}} \neq 0$.

Now we take $A=A_{1} A_{2} \neq 0$, and obtain the identity (3-1). If $u \in M(T)$, by the same discussion, we obtain the lemma. Thus the proof is completed.

Let $\mathrm{Wh}(V)$ denote the set of Whittaker vectors for any Whittaker module $V$. In what follows, we determine the set $\mathrm{Wh}(M)$.

Proposition 3.4. (1) If $h(\epsilon), f(\epsilon)$ act on $M$ as 0 , then $\mathrm{Wh}(M)=M(H)$.

(2) If $h(\epsilon)$ acts on $M$ as 0 and $f(\epsilon)$ does not act as 0 , then $\mathrm{Wh}(M)=M(T)$.

(3) If $h(\epsilon)$ does not act as 0 , then $\mathrm{Wh}(M)=\mathbb{C} v$.

Proof. From Lemma 3.2, we see that any element in $M \backslash M(H)$ is not a Whittaker vector, thus we have $\mathrm{Wh}(M) \subseteq M(H)$.

(1) Suppose $f(\epsilon)=h(\epsilon)=0$ on $M$. For any nonzero element $u \in M(H)$, we prove that $u$ is a Whittaker vector. Write

$$
u=\sum_{i=1}^{n} f_{i} v
$$


where $f_{i}$ are monomials with variables from $\left\{t^{-j \epsilon}, E(-j \epsilon) \mid j \in \mathbb{N}\right\}$. Then for any $j \in \mathbb{N}$, we have

$$
(E(j \epsilon)-\varphi(E(j \epsilon))) u=j \sum_{i=1}^{n}\left(f(\epsilon) \partial_{j}^{\prime}\left(f_{i}\right) v+h(\epsilon) \partial_{j}^{\prime \prime}\left(f_{i}\right) v\right)=0,
$$

where $\partial_{j}^{\prime}$ and $\partial_{j}^{\prime \prime}$ have the same meaning as in the proof of Lemma 3.3. Since $h(\epsilon)=0$, we have $\left[t^{i \epsilon}, E(-j \epsilon)\right]=0$ on $M$ for any $i, j \in \mathbb{N}$, and $t^{i \epsilon}$ commutes with all $f_{k}$ for $1 \leq k \leq n$ on $M$. This implies $t^{i \epsilon} u=\varphi\left(t^{i \epsilon}\right) u$ for all $i \in \mathbb{N}$. Moreover, for all $\alpha \in G_{+} \backslash \mathbb{Z} \epsilon$, note that $E(\alpha) u=\varphi(E(\alpha)) u$, and $t^{\alpha} u=\varphi\left(t^{\alpha}\right) u$ by Lemma 3.1(2). Thus $u \in \mathrm{Wh}(M)$ and we have $\operatorname{Wh}(M)=M(H)$.

(2) Suppose $h(\epsilon)=0, f(\epsilon) \neq 0$ on $M$ and $u \in M(H) \backslash M(T)$, then there exist some $m, p \in \mathbb{N}$ such that

$$
u=\sum_{r=0}^{m} \sum c_{r k n} t^{-k_{s} \epsilon} \cdots t^{-k_{1} \epsilon} E\left(-n_{l} \epsilon\right) \cdots E\left(-n_{1} \epsilon\right)(E(-p \epsilon))^{r} v, \quad c_{r k n} \in \mathbb{C}^{\times},
$$

where the second sum is finite and ranges over $s, l \in \mathbb{Z}_{+}, k_{s} \geq \cdots \geq k_{1} \in \mathbb{N}$, $n_{l} \geq \cdots \geq n_{1} \in \mathbb{N}$, and $n_{1}>p$. Then we have

$$
\begin{aligned}
& (E(p \epsilon)-\varphi(E(p \epsilon))) u \\
& \quad=\sum_{r=0}^{m} \sum c_{r k n}\left[E(p \epsilon), t^{-k_{s} \epsilon} \cdots t^{-k_{1} \epsilon} E\left(-n_{l} \epsilon\right) \cdots E\left(-n_{1} \epsilon\right)(E(-p \epsilon))^{r}\right] v \\
& \quad=p f(\epsilon) \sum_{r=1}^{m} \sum r c_{r k n} t^{-k_{s} \epsilon} \cdots t^{-k_{1} \epsilon} E\left(-n_{l} \epsilon\right) \cdots E\left(-n_{1} \epsilon\right)(E(-p \epsilon))^{r-1} v \neq 0,
\end{aligned}
$$

which implies that $u$ is not a Whittaker vector and $\mathrm{Wh}(M) \subseteq M(T)$. For any $u \in M(T)$, it is easy to check that $u$ is a Whittaker vector as in the discussion in (1).

(3) Suppose $h(\epsilon) \neq 0$ on $M$ and $u \in M(H) \backslash \mathbb{C} v$. Since $h(\epsilon) \neq 0$, Lemma 3.3 shows that $u$ is not a Whittaker vector. Thus $\mathrm{Wh}(M)=\mathbb{C} v$.

\section{Irreducible quotients of the universal Whittaker modules}

In this section we study irreducibility for the universal Whittaker modules and we define a $\mathbb{Z}$-gradation on them, then we determine all $\mathbb{Z}$-graded irreducible quotients for the reducible universal Whittaker modules.

The Lie algebra $L$ has a $\mathbb{Z}$-gradation $L=\bigoplus_{n \in \mathbb{Z}} L(n)$, where

$$
L(-n)= \begin{cases}\bigoplus_{m \in \mathbb{Z}}\left(\mathbb{C} t^{m \epsilon+n \epsilon^{\prime}}+\mathbb{C} E\left(m \epsilon+n \epsilon^{\prime}\right)\right) & \text { if } n \neq 0, \\ \bigoplus_{m \in \mathbb{Z} \backslash\{0\}}\left(\mathbb{C} t^{m \epsilon}+\mathbb{C} E(m \epsilon)\right) \oplus L_{0} & \text { if } n=0 .\end{cases}
$$


Set $B_{H}(0)=\{1\}$, and for $h \in \mathbb{N}$, recall that $B$ is a basis of $U\left(L_{-}\right)$and we set

$$
B_{H}(h)=\left\{X\left(-\alpha_{n}\right) \cdots X\left(-\alpha_{1}\right) \in B \mid n \in \mathbb{N}, \alpha_{i} \in G_{+} \backslash \mathbb{Z} \epsilon, \sum_{i=1}^{n} \alpha_{i}[2]=h\right\} .
$$

Let $M(h)=B_{H}(h) M(H)$ for $h \in \mathbb{Z}_{+}$. We have that $M=\bigoplus_{h \in \mathbb{Z}_{+}} M(h)$ and $L(n) M(h) \subseteq M(n+h)$. Hence $M$ is $\mathbb{Z}$-graded. Note that $M(0)=M(H)=U(H) v$ is a $U(H)$-module.

Recall from (1-2) the definition of $h(\epsilon)$. The following theorem determines when the universal Whittaker module is irreducible.

Theorem 4.1. The universal Whittaker module $M$ is irreducible if and only if $h(\epsilon) \neq 0$.

Proof. Suppose that $h(\epsilon)=0$. By Proposition 3.4(1) and (2), we see that $M$ has a nonzero Whittaker vector $w \notin \mathbb{C} v$. It is easy to see that $U(L) w$ is a proper submodule of $M$.

Conversely, suppose $V$ is a nonzero submodule of $M$, and take $0 \neq w \in V \backslash \mathbb{C} v$. Lemma 3.2 and Lemma 3.3 imply that $h(\epsilon)^{k} v \in \mathcal{U}(L) w \subseteq V$ for some $k \in \mathbb{N}$. Since $h(\epsilon) \neq 0$, we have $v \in V$. Thus $V=M$.

Now we determine all $\mathbb{Z}$-graded irreducible quotients for the universal Whittaker modules on which $h(\epsilon)$ acts as 0 by constructing all maximal $\mathbb{Z}$-graded submodules. The main idea is that we first construct all maximal $U(H)$-submodules of $M(H)$, then we build up maximal $\mathbb{Z}$-graded $U(L)$-submodules of $M$. We divide the construction into two cases: $f(\epsilon)=h(\epsilon)=0$ on $M$, and $f(\epsilon) \neq 0, h(\epsilon)=0$ on $M$. Let $M$ denote the set of all maximal $\mathbb{Z}$-graded $U(L)$-submodules of $M$ and $M_{H}$ denote the set of all maximal $U(H)$-submodules of $M(H)$.

First we consider the case where $f(\epsilon)=h(\epsilon)=0$ on $M$. For any pair

$$
(a, b)=\left(\left(a_{i}\right)_{i \in \mathbb{N}},\left(b_{i}\right)_{i \in \mathbb{N}}\right) \in \mathbb{C}^{\mathbb{N}} \times \mathbb{C}^{\mathbb{N}},
$$

let $I_{a b}$ denote the ideal of $\mathcal{U}\left(H_{-}\right)$generated by $\left\{t^{-i \epsilon}-a_{i}, E(-i \epsilon)-b_{i} \mid i \in \mathbb{N}\right\}$. Clearly $I_{a b}$ is maximal.

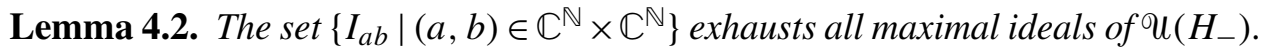

Proof. Suppose $K$ is a maximal ideal of $U\left(H_{-}\right)$. Since $U\left(H_{-}\right)$is an integral domain, $\mathcal{U}\left(H_{-}\right) / K$ is a field extension of $\mathbb{C}$. Notice that any nontrivial field extension of $\mathbb{C}$ is of uncountable dimension over $\mathbb{C}$, but $\mathcal{U}\left(H_{-}\right) / K$ is of countable dimension by the PBW theorem, so $U\left(H_{-}\right) / K \cong \mathbb{C}$. Then we have an algebra epimorphism $\pi$ : $u\left(H_{-}\right) \rightarrow U\left(H_{-}\right) / K \cong \mathbb{C}$ with kernel $K$. Set $a_{i}=\pi\left(t^{-i \epsilon}\right)$ and $b_{i}=\pi(E(-i \epsilon))$ for all $i \in \mathbb{N}$ and $(a, b)=\left(\left(a_{i}\right)_{i \in \mathbb{N}},\left(b_{i}\right)_{i \in \mathbb{N}}\right)$. Clearly, $t^{-i \epsilon}-a_{i}, E(-i \epsilon)-b_{i} \in \operatorname{ker} \pi=K$ for all $i \in \mathbb{N}$. That is, $I_{a b} \subseteq K$. Since $I_{a b}$ is maximal, we have $I_{a b}=K$. 
From Lemma 4.2, we see that any maximal $U\left(H_{-}\right)$-submodule of $M(H)$ is of the form $I_{a b} v$ for some $(a, b) \in \mathbb{C}^{\mathbb{N}} \times \mathbb{C}^{\mathbb{N}}$. Thus $I_{a b} v$ for $(a, b) \in \mathbb{C}^{\mathbb{N}} \times \mathbb{C}^{\mathbb{N}}$ are maximal $U(H)$-submodules of $M(H)$. Furthermore, we claim that any maximal $\mathcal{U}(H)$-submodule of $M(H)$ is of the form $I_{a b} v$ for some $(a, b) \in \mathbb{C}^{\mathbb{N}} \times \mathbb{C}^{\mathbb{N}}$. Indeed, suppose that $V$ is a maximal $U(H)$-submodule of $M(H)$. Then $V$ is a $U\left(H_{-}\right)$submodule of $M(H)$. Thus there exists some $(a, b) \in \mathbb{C}^{\mathbb{N}} \times \mathbb{C}^{\mathbb{N}}$ such that $V \subseteq I_{a b} v$. So $V=I_{a b} v$. That is, $\mathcal{M}_{H}=\left\{I_{a b} v \mid(a, b) \in \mathbb{C}^{\mathbb{N}} \times \mathbb{C}^{\mathbb{N}}\right\}$.

Let $(a, b) \in \mathbb{C}^{\mathbb{N}} \times \mathbb{C}^{\mathbb{N}}$. For $h \in \mathbb{Z}_{+}$, we define

$$
M_{a b}(h)=\left\{u \in M(h) \mid X\left(\epsilon^{\prime}+i_{1} \epsilon\right) \cdots X\left(\epsilon^{\prime}+i_{h} \epsilon\right) u \in I_{a b} v \forall i_{1}, \ldots, i_{h} \in \mathbb{Z}\right\} .
$$

Set $M_{a b}=\sum_{h \in \mathbb{Z}_{+}} M_{a b}(h)$. We claim that $M_{a b}$ is a proper submodule of $M$. Indeed, since $v \notin M_{a b}$, we see that $M_{a b} \varsubsetneqq M$. To prove that $M_{a b}$ is an $L$-submodule of $M$, note that $\left\{X\left( \pm \epsilon^{\prime}+i \epsilon\right) \mid i \in \mathbb{Z}\right\}$ generates $L$, thus we only need to prove the two inclusions

$$
X\left(\epsilon^{\prime}+i \epsilon\right) M_{a b}(h) \subseteq M_{a b}(h-1) \quad \text { and } \quad X\left(-\epsilon^{\prime}+i \epsilon\right) M_{a b}(h) \subseteq M_{a b}(h+1)
$$

for any $i \in \mathbb{Z}$ and $h \in \mathbb{Z}_{+}$. The first one is obvious. For the second one, let $u \in M_{a b}(h)$, and note that for $\alpha \in G_{+} \backslash \mathbb{Z} \epsilon$ we have $X(\alpha) M(H)=0$ by Lemma 3.1. Then for any $i, i_{1}, \ldots, i_{h+1} \in \mathbb{Z}$, we have

$$
\begin{aligned}
X\left(\epsilon^{\prime}+i_{1} \epsilon\right) \cdots X\left(\epsilon^{\prime}+i_{h+1} \epsilon\right) X\left(-\epsilon^{\prime}+i \epsilon\right) u & \\
=X\left(-\epsilon^{\prime}\right. & +i \epsilon) X\left(\epsilon^{\prime}+i_{1} \epsilon\right) \cdots X\left(\epsilon^{\prime}+i_{h+1} \epsilon\right) u \\
& +\left[X\left(\epsilon^{\prime}+i_{1} \epsilon\right) \cdots X\left(\epsilon^{\prime}+i_{h+1} \epsilon\right), X\left(-\epsilon^{\prime}+i \epsilon\right)\right] u \\
\in X\left(-\epsilon^{\prime}+i \epsilon\right) X\left(\epsilon^{\prime}+i_{1} \epsilon\right) I_{a b} v & \\
& +\sum_{k, j_{1}, \cdots, j_{h} \in \mathbb{Z}} \mathbb{C} X(k \epsilon) X\left(\epsilon^{\prime}+j_{1} \epsilon\right) \cdots X\left(\epsilon^{\prime}+j_{h} \epsilon\right) u \\
\subseteq & \sum_{k \in \mathbb{Z}} X(k \epsilon) I_{a b} v \subseteq I_{a b} v
\end{aligned}
$$

where $X(0)=1$. Thus the second inclusion is obtained. Moreover, it is easy to see that $M_{a b}$ is $\mathbb{Z}$-graded.

In what follows, we prove that the $M_{a b}$ for $(a, b) \in \mathbb{C}^{\mathbb{N}} \times \mathbb{C}^{\mathbb{N}}$ exhaust all maximal $\mathbb{Z}$-graded submodules of $M$. The following result gives the characterization of all maximal $\mathbb{Z}$-graded $U(L)$-submodules of $M$ for the case $f(\epsilon)=h(\epsilon)=0$.

Proposition 4.3. $M=\left\{M_{a b} \mid(a, b) \in \mathbb{C}^{\mathbb{N}} \times \mathbb{C}^{\mathbb{N}}\right\}$. Moreover, all $M_{a b}$ for $(a, b) \in$ $\mathbb{C}^{\mathbb{N}} \times \mathbb{C}^{\mathbb{N}}$ are maximal L-submodules of $M$. 
Proof. First we prove that $M_{a b}$ is a maximal $L$-submodule of $M$ for any $(a, b) \in$ $\mathbb{C}^{\mathbb{N}} \times \mathbb{C}^{\mathbb{N}}$. Note that for any $u \in M$, we may write

$$
u=u_{h}+u^{\prime}
$$

for some $h \in \mathbb{Z}_{+}$, where $0 \neq u_{h} \in M(h)$ and $u^{\prime} \in \sum_{h^{\prime}<h} M\left(h^{\prime}\right)$. Then we have

$$
X\left(\epsilon^{\prime}+i_{1} \epsilon\right) \cdots X\left(\epsilon^{\prime}+i_{h} \epsilon\right) u=X\left(\epsilon^{\prime}+i_{1} \epsilon\right) \cdots X\left(\epsilon^{\prime}+i_{h} \epsilon\right) u_{h} \in M(0)=M(H)
$$

for any $i_{1}, \ldots, i_{h} \in \mathbb{Z}$. Now for any $(a, b) \in \mathbb{C}^{\mathbb{N}} \times \mathbb{C}^{\mathbb{N}}$ and $u \in M \backslash M_{a b}$, write $u=u_{h}+u^{\prime}$ as in (4-1). We claim that there exists some $w \in\left(M(H) \backslash I_{a b} v\right) \cap(U(L) u)$. In fact, if $h=0$, then the claim holds for $w=u$. If $h>0$, we may assume that $u_{h} \notin M_{a b}$; otherwise, if $u_{h} \in M_{a b}$, then $u^{\prime}=u-u_{h} \in M \backslash M_{a b}$, thus we may consider $u^{\prime}$ instead of $u$. Then by the definition of $M_{a b}$ and (4-2), we have

$$
X\left(\epsilon^{\prime}+i_{1} \epsilon\right) \cdots X\left(\epsilon^{\prime}+i_{h} \epsilon\right) u=X\left(\epsilon^{\prime}+i_{1} \epsilon\right) \cdots X\left(\epsilon^{\prime}+i_{h} \epsilon\right) u_{h} \in M(H) \backslash I_{a b} v
$$

for some $i_{1}, \ldots, i_{h} \in \mathbb{Z}$. Take $w=X\left(\epsilon^{\prime}+i_{1} \epsilon\right) \cdots X\left(\epsilon^{\prime}+i_{h} \epsilon\right) u$ for $i_{1}, \ldots, i_{h} \in \mathbb{Z}$ satisfying (4-3). Obviously, $w \in\left(M(H) \backslash I_{a b} v\right) \cap(U(L) u)$.

Since $I_{a b} v$ is a maximal $U(H)$-submodule of $M(H)$, we have

$$
v \in M(H)=I_{a b} v+U(H) w \subseteq M_{a b}+U(L) u .
$$

Since $v$ generates $M$, it follows that $M=M_{a b}+\mathcal{U}(L) u$ for any $u \in M \backslash M_{a b}$. Thus $M_{a b}$ is maximal. Since all $M_{a b}$ are $\mathbb{Z}$-graded, we have $M \supseteq\left\{M_{a b} \mid(a, b) \in \mathbb{C}^{\mathbb{N}} \times \mathbb{C}^{\mathbb{N}}\right\}$.

On the other hand, let $N \in \mathcal{M}$. Note that $N \cap M(H)$ is a proper $U(H)$-submodule of $M(H)$. Then there exists $(a, b) \in \mathbb{C}^{\mathbb{N}} \times \mathbb{C}^{\mathbb{N}}$ such that $N \cap M(H) \subseteq I_{a b} v$.

Take any $u \in N$ and write $u=u_{h}+u^{\prime}$ as in (4-1). If $h=0$, we see that $u=u_{0} \in N \cap M(H) \subseteq I_{a b} v \subseteq M_{a b}$. If $h>0$, then we have

$$
X\left(\epsilon^{\prime}+i_{1} \epsilon\right) \cdots X\left(\epsilon^{\prime}+i_{h} \epsilon\right) u=X\left(\epsilon^{\prime}+i_{1} \epsilon\right) \cdots X\left(\epsilon^{\prime}+i_{h} \epsilon\right) u_{h} \in N \cap M(H) \subseteq I_{a b} v .
$$

It follows that $u_{h} \in M_{a b}$. Since $N$ is $\mathbb{Z}$-graded, we have $u_{h} \in N$. So $u^{\prime}=u-u_{h} \in N$. Now by induction on $h$ we get that $u \in M_{a b}$. So $N \subseteq M_{a b}$ and therefore $N=M_{a b}$. This completes the proof.

Now we consider the second case, when $h(\epsilon)=0$ and $f(\epsilon) \neq 0$ on $M$. For any $\xi=\left(\xi_{i}\right)_{i \in \mathbb{N}} \in \mathbb{C}^{\mathbb{N}}$, let $J_{\xi}$ denote the ideal of $\mathcal{U}\left(T_{-}\right)$generated by $\left\{t^{-i \epsilon}-\xi_{i} \mid i \in \mathbb{N}\right\}$. Since $U\left(T_{-}\right)$is commutative, a similar argument as in Lemma 4.2 shows that $\left\{J_{\xi} \mid \xi \in \mathbb{C}^{\mathbb{N}}\right\}$ exhausts all maximal ideals of $\mathcal{U}\left(T_{-}\right)$, so $\left\{J_{\xi} v \mid \xi \in \mathbb{C}^{\mathbb{N}}\right\}$ exhausts all maximal $U\left(T_{-}\right)$-submodules of $U\left(T_{-}\right) v=M(T)$. Note that $M(H)=U(E) M(T)$. We give all maximal $U(H)$-submodules of $M(H)$ in the following proposition.

$$
\mu_{H}=\left\{u(E) J_{\xi} v \mid \xi \in \mathbb{C}^{\mathbb{N}}\right\} .
$$


Proof. Since $h(\epsilon)=0$, we have

$$
T \mathcal{U}(E) J_{\xi} v=U(E) T J_{\xi} v \subseteq \cup(E) J_{\xi} v .
$$

Moreover, for any $k \in \mathbb{Z} \backslash\{0\}$, it is obvious that $E(k \epsilon) \cup(E) J_{\xi} v \subseteq \cup(E) J_{\xi} v$. So $u(E) J_{\xi} v$ is a proper $U(H)$-submodule of $M(H)$. For any $u \in M(H) \backslash u(E) J_{\xi} v$, we may write

$$
u=\sum_{i=1}^{n} a_{i} f_{i} v_{i}, \quad n \in \mathbb{N},
$$

where $a_{i} \neq 0, v_{i} \in U\left(T_{-}\right) v=M(T), f_{i}$ for $1 \leq i \leq n$ are monic monomials with variables from $\{E(-j \epsilon) \mid j \in \mathbb{N}\}$. We remark that, since $u \in M(H) \backslash \mathcal{U}(E) J_{\xi} v$, at least one $v_{i} \notin J_{\xi} v$. Set $J=\left\{i \in\{1, \ldots, n\} \mid v_{i} \notin J_{\xi} v\right\} \neq \varnothing$ and

$$
u^{\prime}=u-\sum_{i \notin J} a_{i} f_{i} v_{i}=\sum_{i \in J} a_{i} f_{i} v_{i} .
$$

Since $f_{i} v_{i} \in U(E) J_{\xi} v$ for $i \notin J$, and since $U(E) J_{\xi} v$ is a $U(H)$-module, it follows that $u(H) u^{\prime} \subseteq \mathcal{U}(H) u+\mathcal{U}(E) J_{\xi} v$. Without loss of generality, we may assume that $1 \in J$ and $f_{1}$ has the maximal degree among $\left\{f_{i} \mid i \in J\right\}$. Write

$$
f_{1}=E(-\epsilon)^{m_{1}} \cdots E(-r \epsilon)^{m_{r}}
$$

for some $m_{i} \in \mathbb{Z}_{+}$. For any monomial $g$ with variables from $\{E(-j \epsilon) \mid j \in \mathbb{N}\}$, note that $[E(i \epsilon), E(-j \epsilon)]=\delta_{i j} i f(\epsilon)$ and $\left[E(i \epsilon), t^{-j \epsilon}\right]=\delta_{i j} i h(\epsilon)$ for any $i, j \in \mathbb{N}$. Thus for any $w \in \mathcal{U}\left(T_{-}\right) v=M(T)$ we have

$$
(E(i \epsilon)-\varphi(E(i \epsilon))) g w=i f(\epsilon) \partial_{i}^{\prime}(g) w,
$$

where $\partial_{i}^{\prime}(g)$ is the partial derivative of $g$ with respect to $E(-i \epsilon)$. Then by induction on $r$, it is easy to see that, for $i \in J$,

$$
(E(\epsilon)-\varphi(E(\epsilon)))^{m_{1}} \cdots(E(r \epsilon)-\varphi(E(r \epsilon)))^{m_{r}} f_{i} v_{i}=\delta_{1, i} \prod_{j=1}^{r} m_{j} !(j f(\epsilon))^{m_{j}} v_{1} .
$$

So we get

$$
(E(\epsilon)-\varphi(E(\epsilon)))^{m_{1}} \cdots(E(r \epsilon)-\varphi(E(r \epsilon)))^{m_{r}} u^{\prime}=A f(\epsilon)^{m_{1}+\cdots+m_{r}} v_{1},
$$

where $A=a_{1} \prod_{j=1}^{r} m_{j} ! j^{m_{j}} \neq 0$. Since $f(\epsilon) \neq 0$, it follows that $v_{1} \in \mathcal{U}(H) u^{\prime} \cap M(T)$.

Since $v_{1} \notin J_{\xi} v$ and $J_{\xi} v$ is a maximal $U\left(T_{-}\right)$-submodule of $M(T)$, we have

$$
v \in M(T)=U\left(T_{-}\right) v_{1}+J_{\xi} v \subseteq U(H) u^{\prime}+\mathcal{U}(E) J_{\xi} v \subseteq U(H) u+U(E) J_{\xi} v .
$$

This implies that $M(H)=\cup(H) u+\mathcal{U}(E) J_{\xi} v$ for any $u \in M(H) \backslash \mathcal{U}(E) J_{\xi} v$, and thus $U(E) J_{\xi} v$ is a maximal $U(H)$-submodule of $M(H)$. That is,

$$
\mathcal{M}_{H} \supseteq\left\{U(E) J_{\xi} v \mid \xi \in \mathbb{C}^{\mathbb{N}}\right\} .
$$


On the other hand, we note that any $\mathcal{U}(H)$-module $W \in \mathcal{M}_{H}$ is also a $\mathcal{U}\left(T_{-}\right)$submodule of $M(H)$. Thus $W \cap M(T)$ is a proper $U\left(T_{-}\right)$-submodule of $M(T)$. It follows that $W \cap M(T) \subseteq J_{\xi} v$ for some $\xi \in \mathbb{C}^{\mathbb{N}}$.

For any nonzero element $u \in W \subseteq M(H)$, we write

$$
u=\sum_{i=1}^{k} g_{i} v_{i}, \quad k \in \mathbb{N},
$$

where $v_{i} \in M(T)$ and $g_{i}$ for $1 \leq i \leq k$ are monomials with variables from $\{E(-j \epsilon) \mid$ $j \in \mathbb{N}\}$. Without loss of generality, we may assume that $g_{1}$ has the maximal degree. If $\operatorname{deg} g_{1}=0$, we have $u \in M(T) \cap W \subseteq J_{\xi} v \subseteq \mathcal{U}(E) J_{\xi} v$ for some $\xi \in \mathbb{C}^{\mathbb{N}}$. If $\operatorname{deg} g_{1}>0$, write

$$
g_{1}=a_{1} E(-\epsilon)^{m_{1}} \cdots E(-r \epsilon)^{m_{r}}
$$

for $a_{1} \in \mathbb{C}^{\times}, m_{i} \in \mathbb{Z}_{+}, i=1, \ldots, r$. Then by induction on $r$ it is easy to see that

$$
(E(\epsilon)-\varphi(E(\epsilon)))^{m_{1}} \cdots(E(r \epsilon)-\varphi(E(r \epsilon)))^{m_{r}} g_{i} v_{i}=\delta_{1, i} a_{1} \prod_{j=1}^{r} m_{j} !(j f(\epsilon))^{m_{j}} v_{1} \text {. }
$$

So we get

$$
(E(\epsilon)-\varphi(E(\epsilon)))^{m_{1}} \cdots(E(r \epsilon)-\varphi(E(r \epsilon)))^{m_{r}} u=A f(\epsilon)^{m_{1}+\cdots+m_{r}} v_{1},
$$

where $A=a_{1} \prod_{j=1}^{r} m_{j} ! j^{m_{j}} \neq 0$. Since $f(\epsilon) \neq 0$, we have $v_{1} \in \mathcal{U}(E) u \subseteq W$. Thus $v_{1} \in W \cap M(T) \subseteq J_{\xi} v$ for some $\xi \in \mathbb{C}^{\mathbb{N}}$. It follows that $g_{1} v_{1} \in W \cap \mathcal{U}(E) J_{\xi} v$. So $u-g_{1} v_{1} \in W$. By iteration, we can get $u \in W \cap U(E) J_{\xi} v \subseteq U(E) J_{\xi} v$. Thus $W \subseteq U(E) J_{\xi} v$. Then, by the maximality of $W$ as a $U(H)$-submodule of $M(H)$ and since $U(E) J_{\xi} v$ is a proper $U(H)$-submodule of $M(H)$, we have $W=U(E) J_{\xi} v$. Thus $\mathcal{M}_{H} \subseteq\left\{U(E) J_{\xi} v \mid \xi \in \mathbb{C}^{\mathbb{N}}\right\}$. This completes the proof.

In what follows, we construct certain submodules of $M$, and prove that these submodules exhaust all the maximal $\mathbb{Z}$-graded submodules of $M$ in the case that $h(\epsilon)=0, f(\epsilon) \neq 0$ on $M$.

For $\xi \in \mathbb{C}^{\mathbb{N}}$, let $M_{\xi}(0)=U(E) J_{\xi} v$. For any $h \in \mathbb{N}$, set

$$
M_{\xi}(h)=\left\{u \in M(h) \mid X\left(\epsilon^{\prime}+i_{1} \epsilon\right) \cdots X\left(\epsilon^{\prime}+i_{h} \epsilon\right) u \in \mathcal{U}(E) J_{\xi} v \forall i_{1}, \ldots, i_{h} \in \mathbb{Z}\right\} .
$$

Set $M_{\xi}=\bigoplus_{h \in \mathbb{Z}_{+}} M_{\xi}(h)$. By a similar argument as in the previous case where $f(\epsilon)=h(\epsilon)=0$ on $M$, we can prove that $M_{\xi}$ is a proper $\mathbb{Z}$-graded $L$-submodule of $M$. Then by a similar proof as in Proposition 4.3, we obtain the following result.

Proposition 4.5. $M=\left\{M_{\xi} \mid \xi \in \mathbb{C}^{\mathbb{N}}\right\}$. Moreover, for any $\xi \in \mathbb{C}^{\mathbb{N}}, M_{\xi}$ is a maximal $L$-submodule of $M$.

By Theorem 4.1, Proposition 4.3 and Proposition 4.5, we obtain the main result of the paper. 
Theorem 4.6. Suppose that $V$ is a $\mathbb{Z}$-graded irreducible quotient of the universal Whittaker module $M_{\varphi, k_{1}, k_{2}, k_{3}, k_{4}}$ of type $\left(\varphi, k_{1}, k_{2}, k_{3}, k_{4}\right)$ over L. Then $V$ is irreducible as an L-module. Furthermore:

(1) If $h(\epsilon) \neq 0$ on $V$, then $V=M_{\varphi, k_{1}, k_{2}, k_{3}, k_{4}}$.

(2) If $h(\epsilon)=f(\epsilon)=0$ on $V$, then

$$
V=V_{a b}=: M_{\varphi, k_{1}, k_{2}, k_{3}, k_{4}} / M_{a b}
$$

for some $(a, b) \in \mathbb{C}^{\mathbb{N}} \times \mathbb{C}^{\mathbb{N}}$. Moreover, $V_{a b} \cong V_{a^{\prime} b^{\prime}}$ if and only if $(a, b)=\left(a^{\prime}, b^{\prime}\right)$.

(3) If $h(\epsilon)=0, f(\epsilon) \neq 0$ on $V$, then

$$
V=V_{\xi}=: M_{\varphi, k_{1}, k_{2}, k_{3}, k_{4}} / M_{\xi}
$$

for some $\xi \in \mathbb{C}^{\mathbb{N}}$. Moreover, $V_{\xi} \cong V_{\xi^{\prime}}$ if and only if $\xi=\xi^{\prime}$.

Remark. For the Virasoro-like algebra $\mathscr{V}$, the notion of Whittaker module of type $\left(\varphi, k_{3}, k_{4}\right)$ was given in [Guo and Liu 2011b]. We can similarly define a $\mathbb{Z}$-gradation on the universal Whittaker module $M_{\varphi, k_{3}, k_{4}}$. For any $\alpha \in \mathbb{Z}^{2} \backslash\{(0,0)\}$, we define the action of $t^{\alpha}$ on $M_{\varphi, k_{3}, k_{4}}$ trivially; then it is easy to see that $K_{1}, K_{2}$, hence $h(\epsilon)$, act as 0 on $M_{\varphi, k_{3}, k_{4}}$, and $M_{\varphi, k_{3}, k_{4}}$ becomes a Whittaker module of type $\left(\varphi, 0,0, k_{3}, k_{4}\right)$ for $L$. Therefore, Theorem 4.6 gives all $\mathbb{Z}$-graded irreducible quotients of the universal Whittaker modules for $\mathscr{V}$ and also proves that all $\mathbb{Z}$-graded irreducible quotients of the universal Whittaker modules are actually irreducible.

Furthermore, we prove that any $\mathbb{Z}$-graded irreducible quotient of a universal Whittaker module for $L$ admits a unique Whittaker vector up to scalars. This result also applies to the Virasoro-like algebra.

Corollary 4.7. Suppose that $V$ is a $\mathbb{Z}$-graded irreducible quotient of a universal Whittaker module. Then $\operatorname{dim} \mathrm{Wh}(V)=1$.

Proof. Using Theorem 4.6, we prove this corollary in three cases.

Case 1: $h(\epsilon) \neq 0$ on $V$. Notice that $V=M$ and $\mathrm{Wh}(M)=\mathbb{C} v$. So $\operatorname{dim} \mathrm{Wh}(V)=1$.

Case 2: $h(\epsilon)=f(\epsilon)=0$ on $V$. We have $V=M / M_{a b}$ for some $(a, b) \in \mathbb{C}^{\mathbb{N}} \times \mathbb{C}^{\mathbb{N}}$. Let $u+M_{a b}$ be a Whittaker vector for some $u \in M \backslash M_{a b}$. Write $u=u_{h}+u^{\prime}$, where $0 \neq u_{h} \in M(h)$ and $u^{\prime} \in \sum_{i<h} M(i)$. If $u_{h} \in M_{a b}$, then $u^{\prime}+M_{a b}=u+M_{a b}$. We consider $u^{\prime}$ instead. Hence we may assume that $h$ is the smallest nonnegative integer such that $u_{h} \notin M_{a b}$. We claim that $h=0$.

On the contrary, suppose that $h>0$. Note that $u_{h} \notin M_{a b}$. By the definition of $M_{a b}$ we have

$$
X\left(\epsilon^{\prime}+i_{1} \epsilon\right) \cdots X\left(\epsilon^{\prime}+i_{h} \epsilon\right) u_{h} \in M(H) \backslash I_{a b} v \text { for some } i_{1}, \ldots, i_{h} \in \mathbb{Z} .
$$


So, by Proposition 2.2(2) and (4-3), we have

$$
\begin{gathered}
\left(X\left(\epsilon^{\prime}+i_{1} \epsilon\right)-\varphi\left(X\left(\epsilon^{\prime}+i_{1} \epsilon\right)\right)\right) \cdots\left(X\left(\epsilon^{\prime}+i_{h} \epsilon\right)-\varphi\left(X\left(\epsilon^{\prime}+i_{h} \epsilon\right)\right)\right) u \\
=X\left(\epsilon^{\prime}+i_{1} \epsilon\right) \cdots X\left(\epsilon^{\prime}+i_{h} \epsilon\right) u \\
=X\left(\epsilon^{\prime}+i_{1} \epsilon\right) \cdots X\left(\epsilon^{\prime}+i_{h} \epsilon\right) u_{h} \notin M_{a b} .
\end{gathered}
$$

This contradicts that $u+M_{a b}$ is a Whittaker vector, so $h=0$. Therefore $u \in M(H)$. Since $M(H)=\mathbb{C} v+I_{a b} v$, we have $u \in \mathbb{C} v+M_{a b}$. So

$$
\operatorname{dim} \operatorname{Wh}(V)=\operatorname{dim} \operatorname{Wh}\left(M / M_{a b}\right)=1 .
$$

Case 3: $h(\epsilon)=0, f(\epsilon) \neq 0$ on $V$. The proof is similar to that of Case 2 .

\section{References}

[Arbarello et al. 1988] E. Arbarello, C. De Concini, V. G. Kac, and C. Procesi, "Moduli spaces of curves and representation theory", Comm. Math. Phys. 117:1 (1988), 1-36. MR 89i:14019 Zbl 0647.17010

[Arnal and Pinczon 1974] D. Arnal and G. Pinczon, "On algebraically irreducible representations of the Lie algebra sl(2)", J. Mathematical Phys. 15 (1974), 350-359. MR 50 \#9995 Zbl 0298.17003

[Batra and Mazorchuk 2011] P. Batra and V. Mazorchuk, "Blocks and modules for Whittaker pairs", J. Pure Appl. Algebra 215:7 (2011), 1552-1568. MR 2012c:17013 Zbl 1228.17008

[Benkart and Ondrus 2009] G. Benkart and M. Ondrus, "Whittaker modules for generalized Weyl algebras", Represent. Theory 13 (2009), 141-164. MR 2010e:16039 Zbl 1251.16020

[Block 1981] R. E. Block, "The irreducible representations of the Lie algebra sl(2) and of the Weyl algebra", Adv. in Math. 39:1 (1981), 69-110. MR 83c:17010 Zbl 0454.17005

[Christodoulopoulou 2008] K. Christodoulopoulou, "Whittaker modules for Heisenberg algebras and imaginary Whittaker modules for affine Lie algebras", J. Algebra 320:7 (2008), 2871-2890. MR 2009i:17009 Zbl 1221.17009

[Guo and Liu 2011a] X. Guo and X. Liu, "Whittaker modules over generalized Virasoro algebras", Comm. Algebra 39:9 (2011), 3222-3231. MR 2012m:17038 Zbl 1255.17005

[Guo and Liu 2011b] X. Guo and X. Liu, "Whittaker modules over Virasoro-like algebra”, J. Math. Phys. 52:9 (2011), 093504. MR 2012j:17032 Zbl 1272.17028

[Kirkman et al. 1994] E. Kirkman, C. Procesi, and L. Small, "A $q$-analog for the Virasoro algebra", Comm. Algebra 22:10 (1994), 3755-3774. MR 96b:17016 Zbl 0813.17009

[Kostant 1978] B. Kostant, “On Whittaker vectors and representation theory”, Invent. Math. 48:2 (1978), 101-184. MR 80b:22020 Zbl 0405.22013

[Lu and Zhao 2013] R. Lu and K. Zhao, "Generalized oscillator representations of the twisted Heisenberg-Virasoro algebra”, preprint, 2013. arXiv 1308.6023v1

[Ondrus and Wiesner 2009] M. Ondrus and E. Wiesner, "Whittaker modules for the Virasoro algebra", J. Algebra Appl. 8:3 (2009), 363-377. MR 2010f:17040 Zbl 1220.17019

[Xue et al. 2006] M. Xue, W. Lin, and S. Tan, "Central extension, derivations and automorphism group for Lie algebras arising from the 2-dimensional torus”, J. Lie Theory 16:1 (2006), 139-153. MR 2006i:17038 Zbl 1105.17006

[Zhang et al. 2010] X. Zhang, S. Tan, and H. Lian, "Whittaker modules for the Schrödinger-Witt algebra”, J. Math. Phys. 51:8 (2010), 083524. MR 2011m:81152 
Received December 7, 2013.

SHAOBIN TAN

SCHOOL OF MATHEMATICAL SCIENCES

XIAMEN UNIVERSITY

XIAMEN 361005

CHINA

tans@xmu.edu.cn

QING WANG

SCHOOL OF Mathematical SCIENCES

Xiamen University

XIAMEN 361005

CHINA

qingwang@xmu.edu.cn

Chengkang Xu

SCHOol of Mathematical Sciences

XiAMEN UNIVERSITY

XIAMEN 361005

CHINA

xiaoxiongxu@126.com 


\title{
PACIFIC JOURNAL OF MATHEMATICS
}

\author{
msp.org/pjm
}

Founded in 1951 by E. F. Beckenbach (1906-1982) and F. Wolf (1904-1989)

\section{EDITORS}

Don Blasius (Managing Editor)

Department of Mathematics

University of California

Los Angeles, CA 90095-1555

blasius@math.ucla.edu

\author{
Paul Balmer \\ Department of Mathematics \\ University of California \\ Los Angeles, CA 90095-1555 \\ balmer@math.ucla.edu \\ Robert Finn \\ Department of Mathematics \\ Stanford University \\ Stanford, CA 94305-2125 \\ finn@math.stanford.edu \\ Sorin Popa \\ Department of Mathematics \\ University of California \\ Los Angeles, CA 90095-1555 \\ popa@math.ucla.edu
}

\author{
Vyjayanthi Chari \\ Department of Mathematics \\ University of California \\ Riverside, CA 92521-0135 \\ chari@math.ucr.edu \\ Kefeng Liu \\ Department of Mathematics \\ University of California \\ Los Angeles, CA 90095-1555 \\ liu@math.ucla.edu \\ Jie Qing \\ Department of Mathematics \\ University of California \\ Santa Cruz, CA 95064 \\ qing@ cats.ucsc.edu
}

\section{PRODUCTION}

Silvio Levy, Scientific Editor, production@msp.org

\section{SUPPORTING INSTITUTIONS}

ACADEMIA SINICA, TAIPEI

CALIFORNIA INST. OF TECHNOLOGY

INST. DE MATEMÁTICA PURA E APLICADA

KEIO UNIVERSITY

MATH. SCIENCES RESEARCH INSTITUTE

NEW MEXICO STATE UNIV.

OREGON STATE UNIV.

\author{
STANFORD UNIVERSITY \\ UNIV. OF BRITISH COLUMBIA \\ UNIV. OF CALIFORNIA, BERKELEY \\ UNIV. OF CALIFORNIA, DAVIS \\ UNIV. OF CALIFORNIA, LOS ANGELES \\ UNIV. OF CALIFORNIA, RIVERSIDE \\ UNIV. OF CALIFORNIA, SAN DIEGO \\ UNIV. OF CALIF., SANTA BARBARA
}

\author{
Daryl Cooper \\ Department of Mathematics \\ University of California \\ Santa Barbara, CA 93106-3080 \\ cooper@math.ucsb.edu \\ Jiang-Hua Lu \\ Department of Mathematics \\ The University of Hong Kong \\ Pokfulam Rd., Hong Kong \\ jhlu@maths.hku.hk \\ Paul Yang \\ Department of Mathematics \\ Princeton University \\ Princeton NJ 08544-1000 \\ yang@math.princeton.edu
}

These supporting institutions contribute to the cost of publication of this Journal, but they are not owners or publishers and have no responsibility for its contents or policies.

See inside back cover or msp.org/pjm for submission instructions.

The subscription price for 2015 is US \$420/year for the electronic version, and \$570/year for print and electronic.

Subscriptions, requests for back issues and changes of subscribers address should be sent to Pacific Journal of Mathematics, P.O. Box 4163, Berkeley, CA 94704-0163, U.S.A. The Pacific Journal of Mathematics is indexed by Mathematical Reviews, Zentralblatt MATH, PASCAL CNRS Index, Referativnyi Zhurnal, Current Mathematical Publications and Web of Knowledge (Science Citation Index).

The Pacific Journal of Mathematics (ISSN 0030-8730) at the University of California, c/o Department of Mathematics, 798 Evans Hall \#3840, Berkeley, CA 94720-3840, is published twelve times a year. Periodical rate postage paid at Berkeley, CA 94704, and additional mailing offices. POSTMASTER: send address changes to Pacific Journal of Mathematics, P.O. Box 4163, Berkeley, CA 94704-0163.

PJM peer review and production are managed by EditFLOW ${ }^{\circledR}$ from Mathematical Sciences Publishers.

\section{PUBLISHED BY}

\section{mathematical sciences publishers \\ nonprofit scientific publishing}

http://msp.org/

(C) 2015 Mathematical Sciences Publishers 


\section{PACIFIC JOURNAL OF MATHEMATICS}

Volume $273 \quad$ No. $1 \quad$ January 2015

Maximal estimates for Schrödinger equations with inverse-square potential 1

ChangXing MiaO, JUNYong Zhang and JiQiang Zheng

Vassiliev Invariants of Virtual Legendrian Knots

PATRICIA CAHN and ASA LEVI

Some results on the generic vanishing of Koszul cohomology via

deformation theory

JIE WANG

Conformal metrics with constant curvature one and finitely many conical

singularities on compact Riemann surfaces

QING Chen, WeI WANG, Yingyi Wu and BIN XU

$\mathbb{Q}$-bases of the Néron-Severi groups of certain elliptic surfaces

MASAMICHI KURODA

On a prime zeta function of a graph

TAKehiro Hasegawa and SEIKEN SAIto

On Whittaker modules for a Lie algebra arising from the 2-dimensional torus 147

SHaObin TAN, QING WANG and ChENGKANG XU

Fréchet quantum supergroups

AXEL DE GOURSAC

Generators of the Gauss-Picard modular group in three complex dimensions

BaoHua Xie, JieYan Wang and YuePing Jiang

Complete characterization of isolated homogeneous hypersurface singularities

STEPHEN YAu and HuAiQING ZuO

A theorem of Mœglin and Waldspurger for covering groups

SHIV PRAKASH PATEL

Spanning trees and random walks on weighted graphs 